\title{
Gentamicin C1a
}

National Cancer Institute

\section{Source}

National Cancer Institute. Gentamicin C1a. NCI Thesaurus. Code C76149.

One of the major components of the gentamicin complex. Gentamicin C1a lacks methyl groups on the 2-amino-hexose ring and has a free amine at the 6 ' position. 